\title{
Knowledge, Attitude and Practice of Hepatitis B among Informal Caregivers at Ndola Teaching Hospital
}

\author{
Killard SC* \\ Copperbelt University, MicHeal CHilu fva data-8cHool of \\ Medicine, Ndola, Zambia \\ *Correspondling author: Siamunyanga C Killard, \\ Copperbelt University, MicHeal CHilu fva data-8cHool of \\ Medicine, Ndola, Zambia
}

Received: April 13, 2021; Accepted: May 04, 2021;

Published: May 11, 2021

\begin{abstract}
Introduction: Hepatitis B is a highly contagious infection. It is 50 to 100 times more infectious than HIV. It is the world's most common liver infection, which is caused by a DNA-virus, the hepatitis B virus and is transmitted between people through blood, semen, vaginal fluids and mucous membranes. Basic knowledge about hepatitis $B$ is necessary; with more knowledge people take preventive actions against hepatitis B, such as using stick proof cannulas. The general objective of this study was to assess the knowledge, attitude and practice of HBV infection among informal caregivers at Ndola Teaching Hospital, Ndola, Zambia.
\end{abstract}

Method: A Cross sectional study was carried out at Ndola Teaching Hospital from June 2020 to September 2020. Systematic random sampling was used using questionnaire based interviews to consenting informal caregivers above the age of 18 years who were waiting for their patients in Gynecological ward, surgical wards and internal medicine wards.

Results: A total number of 165 informal caregivers at Ndola Teaching Hospital were interviewed using a pretested questionnaire. The highest age group (44.2\%) interviewed was between 25 to 34 years of age. The majority of informal caregivers (52.1\%) went up to secondary education level and $63.0 \%$ of the informal caregivers were females. Most informal caregivers (53.3\%) only had an average knowledge about hepatitis B infection. Informal caregiver's education level was directly associated with the level of knowledge about Hepatitis B ( $p$-value $<0.001)$. Majority $(87.3 \%)$ of the informal caregivers had good attitude towards hepatitis B. There was a direct association ( $p$ value $<0.001$ ) between informal caregiver's knowledge on hepatitis $B$ with their attitude. All the informal caregivers (100\%) had bad practices on Hepatitis B and majority of the respondents $159(96.4 \%)$ were not vaccinated only $6(3.6 \%)$ were vaccinated against HBV.

Conclusion: Hepatitis B is a major health problem globally casting an enormous burden on the health-care system a major source of patient's misery. This illness or disease is preventable. In this study, the majority of the participants had good attitudes making knowledge as the main determinant of their practices. Therefore, primary prevention approach be taken by extensively and frequently teaching all informal care givers in all hospitals and clinics on how to protect themselves from the disease. Pictorial Posters depicting the proper way of handling patients by informal caregivers be displayed in health centers (wards and OPDs).

Keywords: Hepatitis B; Informal caregivers; Knowledge; Attitude; practices

\section{Abbreviation}

AIDS: Acquired Immune Deficiency Syndrome; EPI:

Expanded Program on Immunization; HBcAg: Hepatitis B core antigen; HBM: Health Belief Model; HBsAg: Hepatitis B Surface Antigen; HBV: Hepatitis B Virus; HCC: Hepatocellular Carcinoma; HIV: Human Immunodeficiency Virus; KAP: Knowledge, Attitude and Practice; SPSS: Statistical Package for Social Sciences; TDRC: Tropical Diseases Research Centre; WHO: World Health Organization; ZAMPHIA: Zambia Population Based HIV impact Assessment

\section{Introduction}

\section{Background}

According to the world health Organization [1], hepatitis B is the world's most common liver infection, which is caused by a DNAvirus, the Hepatitis B Virus (HBV). The virus is highly contagious, 50 to 100 times more infectious than HIV, and is transmitted between people through blood, semen, vaginal fluids and mucous membranes. There are more than 2 billion people world-wide, having evidence of recent or past $\mathrm{HBV}$ infection and 350 million are chronic carriers. HBV is unique compared to other sexually transmitted diseases, because it can be prevented with vaccine [1]. In 1964, it became possible to identify people with $\mathrm{HBV}$ using serological testing, searching for hepatitis B surface antigen (HBsAg) [2]. 
Hepatitis B Virus (HBV) is a small prototype DNA virus of the Hepadnaviridae family with features similar to retroviruses. It is partially double-stranded and is classified into eight genotypes, A to $\mathrm{H}$, with each having a distinct geographic distribution [3]. Three types of viral particles are visualized in infectious serum by electron microscopy and the infectious HBV virion known as the "Dane particle", consist of a lipid envelope containing hepatitis B surface antigen (HBsAg) that surrounds an inner nucleocapsid composed of hepatitis B core antigen ( $\mathrm{HBcAg}$ ) [3]. HBV attacks the liver and can cause both acute and chronic infections such as cirrhosis and Hepatocellular Carcinoma (HCC). HBV can persist in infected hepatocytes and has evolved elaborate strategies to evade the immune system, replicating via reverse transcription [4]. Drugs licensed for inhibition of Human Immune Virus (HIV) reverse transcriptase lower the viral load of chronic HBV patients, but they do not cure the infection. However, it can be prevented via currently available safe and effective vaccines which induces protective antibody levels in $>95 \%$ of infants, children and young adults [5].

The hepatitis B virus can survive outside the body for at least 7 days. During this time, the virus can still cause infection if it enters the body of a person who is not protected by the vaccine. The incubation period of the hepatitis B virus is 75 days on average, but can vary from 30 to 180 days. The virus may be detected within 30 to 60 days after infection and can persist and develop into chronic hepatitis B.

In highly endemic areas, hepatitis B is most commonly spread from mother to child at birth (perinatal transmission), or through horizontal transmission (exposure to infected blood), especially from an infected child to an uninfected child during the first 5 years of life. The development of chronic infection is very common in infants infected from their mothers or before the age of 5 years. It is also spread by percutaneous or mucosal exposure to infected blood and various body fluids, as well as through saliva, menstrual, vaginal, and seminal fluids. Sexual transmission of hepatitis B may occur, particularly in unvaccinated men who have sex with men and heterosexual persons with multiple sex partners or contact with sex workers. Infection in adulthood leads to chronic hepatitis in less than $5 \%$ of cases. Transmission of the virus may also occur through the reuse of needles and syringes either in health-care settings or among persons who inject drugs. In addition, infection can occur during medical, surgical and dental procedures, through tattooing, or through the use of razors and similar objects that are contaminated with infected blood [6].

If hepatitis is occurred that is usually chronic (hepatitis B), may not have symptoms in the beginning. Symptoms may not occur until liver damage occurs. Signs and symptoms of acute hepatitis appear quickly. They include: fatigue, flu-like symptoms, dark urine, pale stool and abdominal pain, Loss of appetite, unexplained weight loss, Yellow skin and eyes, which may be signs of jaundice. Since chronic hepatitis develops slowly, these signs and symptoms may be too subtle to notice [7].

The likelihood that infection becomes chronic depends upon the age at which a person becomes infected. Children less than 6 years of age who become infected with the hepatitis B virus are the most likely to develop chronic infections.

In infants and children; 80-90\% of infants infected during the first year of life develop chronic infections and 30-50\% of children infected before the age of 6 years develop chronic infections.

In adults; Less than $5 \%$ of healthy persons who are infected as adults will develop chronic infection and $20-30 \%$ of adults who are chronically infected will develop cirrhosis and/or liver cancer [6].

Therefore, the main aim of this research is to collect basic information on knowledge, attitudes and practices of informal caregivers regarding prevention of $\mathrm{HBV}$ infection as they take care of their patients.

\section{Problem statement}

According to the world health organization [6], Hepatitis B is highly contagious viral infection affecting more than 10 times the number of people infected with HIV. The virus is transmitted through contact with blood or other body fluids of an infected person. The infection attacks the liver and can cause both acute and chronic disease. Although most people are able to clear the virus, 8 to $10 \%$ of adults go on to develop chronic infection. In some individuals, especially among those with weak immune systems, the infection may develop into a severe life - threatening form of acute hepatitis. Chronic Hepatitis B infection, lasting longer than 6 months, may lead to liver cirrhosis, liver failure and liver cancer [6].

In Zambia, the global prevalence of Hepatitis B Virus (HBV) infection was estimated at $3.5 \%$ with about 352 million persons living with chronic $\mathrm{HBV}$ infection and about 1.4 million people dying each year from the disease [6] An estimated 337,454 die due to liver cancer, 462690 due to cirrhosis, and 87,076 due to acute hepatitis. It is estimated that only $5 \%$ of people with chronic HBV infection are aware of their infection status and less than $1 \%$ have access to treatment. In most individuals, both acute and chronic infection develops in adulthood. In Zambia, the estimated overall prevalence of hepatitis B virus is 5.6\% among ages 15-59; prevalence among HIV positive individual is $7.1 \%$ whereas in the HIV negatives it stands at 5.4\% [8]. Hepatitis B virus is fully preventable with effective vaccines [6]. However, in Zambia, the control strategies have focused on blood bank screening and childhood vaccination. Existing hepatitis immunization program in Zambia focusses primarily on children six weeks of age, leaving the adult population at risk. The EPI manual of 2017. Zambia has adopted the WHO goal of eliminating Hepatitis $B$ virus infection by the year 2030 [9]. Recent efforts to address the hepatitis B virus burden have seen the inclusion of hepatitis treatment in the HIV treatment guidelines, but no deliberate efforts have been made to address the preventive measures on the part of the informal caregivers as they take care of their patients as this is one of the population group at risk. Prevention is considered as one best way to safeguard populations' health. Prevention against any disease is proportional to knowledge, attitude and practice of the population and is reflective of the importance that is paid to health related issues by the society. Therefore, the current study aimed at assessing knowledge, attitude and practices of HBV among informal caregivers at Ndola Teaching hospital.

\section{Literature review}

Brief history of hepatitis B virus: The hepatitis B virus was discovered in 1965 when Blumberg and co - workers found the hepatitis B surface antigen, which was originally called the Australia 
antigen because it was found in serum from an Australian patient [10]. Dr. Baruch Sanuel Blumberg was awarded the 1976 Noble prize in physiology or medicine for this discovery. The virus was fully described in the 1970s [11]. In recent times, the rapid and continuous discoveries of the viral disease around the whole world have improved our understanding of the complexity of this unusual virus. Although there has not been any substantial decrease in the overall prevalence of $\mathrm{HBV}$, there is the hope that the next generation will see a decrease in the overall prevalence of $\mathrm{HBV}$, worldwide carrier rate and the incidence of new $\mathrm{HBV}$ infections if current $\mathrm{HBV}$ vaccinations are intensified.

Global situation of hepatitis B: Despite the fact that since 1982 there is a vaccine against HBV that gives $90-100 \%$ protection against infection, there are in the world today more than 325 million people living with chronic hepatitis $\mathrm{B}$. The consequences of this is approximately 1.4 million HBV related deaths every year around the world, where the cause is primary liver cirrhosis or liver cancer [6] The virus is transmitted differently between geographic regions and countries depending on how endemic the HBV is there. In regions where the endemicity is low, it is more common that the virus is transmitted through horizontal routes such as injecting drug use, high - risk sexual behavior and receiving blood products. When in regions with high endemicity, for example in Vietnam, HBV is primarily spread by vertical transmission early in childhood or prenatally, from mother to child at birth [12].

In the United States (U.S.), approximately 1.4 million residents are chronically infected with HBV [2]. According to the fact that during the years 1974-2008, 17.6 million people born in countries of intermediate or high prevalence of chronic hepatitis B have immigrated to U.S., there is an increased burden of chronic hepatitis $B$ in the country. More than half of the estimated chronic hepatitis B cases were from the western pacific region, from countries such as the Philippines, China and Vietnam. These were the main countries of birth for imported cases of chronic hepatitis B. Africa was the second largest region for imported cases of chronic hepatitis B. according to systematic review [13] migrants from East Asia, the Pacific and Sub - Saharan Africa represented a high sero prevalence of chronic hepatitis B, 10.3-11.3 \%, and migrants from Eastern Europe, Central Africa and South Asia were an intermediate sero prevalence. The sero prevalence of chronic hepatitis B was low among migrants from the Caribbean, Latin American, the Middle East and North Africa, Refugees and asylum seekers and higher sero prevalence of chronic hepatitis B compared to migrants.

Hepatitis B-vaccine: Since 1982 there is a vaccine against HBV that gives $95 \%$ protection against the infection [1]. The World Health Organization recommends that all infants should get the HBVvaccination, and their first dose within 24 hours after birth, followed by two more doses to complete the series. The protection lasts for 20 years and is possibly life-long [14].

The hepatitis B vaccine is counted as one of the safest vaccinations. People cannot get HBV from the vaccine and the most common side effects is soreness and redness in the arm where the injection was given (3-29\%) and fever over $37.7^{\circ} \mathrm{C}(1-6 \%)$ [15]. The vaccine is the first "anti-cancer vaccine" because it protects from getting hepatitis $\mathrm{B}$ that is the main source for liver cancer. About $80 \%$ of all liver cancer cases are developed from HBV [16].

A study about the vaccination achievements for the last three decades has been made by Marinho, Meireles \& van Damme [17]. Over the past 30 years investments in the primary prevention have been done to increase the coverage of the universal vaccination programs with great result. In the eighties the HBV vaccine was only given to persons with a great risk of getting the infection but today it is different. Today 179 countries in the world have vaccination against the infection in their routine vaccination program and are given to all infants. According to Marinho et al. [17] the vaccination has proven to give good protection and it is a safe and effective way to prevent populations from developing acute or chronic hepatitis B.

The current vaccination has an efficacy over $90 \%$, after the complete treatment with three doses. The vaccine can be used against all HBV genotypes and serotypes. According to Marinho, there are still big challenges to deal with, such as the occurrence of breakthrough infections, the effectiveness of the universal HBV vaccination and the effect of natural boosting. Marinho et al. [17].

Knowledge of hepatitis B infection: A cross-sectional study by Bui et al [18] investigated Vietnamese Americans knowledge and the prevalence of hepatitis $B$. The results showed that there was a lack of knowledge about HBV, 32.9\% thought incorrectly that HBV was transmitted through casual contacts or by contaminated air or food. The results also showed that there was a lack of knowledge regarding the treatment, $42.7 \%$ knew that there was treatment for HBV and $50.4 \%$ believed that a positive attitude could prevent HBV. The authors of the study discussed about herbal/alternative medication as a part of the Asian culture, that the Vietnamese Americans have not yet been influenced by Western culture and medicine.

A cross- sectional study by Gürakar, Idilman, Keskin and Malik [19] was conducted in Turkey. The aim of the study was to investigate the public awareness about hepatitis B in Turkey and to use the result as a model over the universal effectiveness in health care policy. A questionnaire including 32 questions about awareness of HBV infections, ways of transmission and consequences after getting the infection was used. The Results showed that $60 \%$ of the participants had basic knowledge that hepatitis B was a transmissible infection and $\mathrm{HBV}$ was a major cause of serious liver disease. Promoting awareness of HBV should be a useful and effective strategy to prevent the spread of HBV, as well as it being a cost-effective strategy to use globally.

A cross-sectional study conducted in Lao investigated students in different health professions' knowledge about hepatitis B and the vaccine [20]. In this survey 961 respondents answered demographic questions, if they had been vaccinated and reasons if they were not vaccinated. The results showed that $53 \%$ of the students were not vaccinated. The reason of not getting vaccinated was that they did not know where to get vaccinated from or that the vaccine was too expensive. The study also showed that those who were vaccinated were mostly postgraduate students. The authors made a conclusion that postgraduate students had more knowledge about the HBV infection and therefore had a more preventive attitude. Birungi, Korchmaros, Prescott and Ybarra (2015) also described that information about prevention about infections/certain states should be given to a younger population. 
According to a study Mursy et al, [21], on Knowledge, attitude and practice towards hepatitis $\mathrm{B}$ infection among nurses and midwives in two maternal hospitals in Khartoum, Sudan. The study findings revealed that the percentage of respondents with average knowledge regarding HBV was $58.2 \%$. According to study results, HBV was recognized as a viral infection by overwhelming majority of the respondents (99.9\%). The findings of the study posited that the level of knowledge was not significantly associated with age, occupation, marital status, educational level, or working experience.

Attitude and practices towards hepatitis B infection: In a study by Mursy et al, [21], the great majority of the respondents (86.4\%) showed a favorable attitude towards the preventive measures of HBV infection (instruments sterilization, wearing gloves, $\mathrm{HBV}$ vaccination and PEP). More than half of the participants (64.5\% believed that instruments sterilization is important to prevent transmission, and $72.7 \%$ of them think that wearing gloves is important to prevent transmission. Among the respondents, 65.5\% of them were considered to have a safe practice regarding HBV infection. According to the study findings, a vast majority of the participants always used sterilized instruments (90\%) and wore gloves (90.9\%).

A cross sectional study on knowledge, attitude, and practice towards hepatitis B virus among pregnant women attending antenatal care at the University of Gondar Comprehensive Specialized Hospital, Northwest Ethiopia was done by Gebrecherkos et al. [22]. The study showed that lack of Knowledge may influence the attitudes of the mother towards interventions that could reduce the risk of transmission to their infants. Of the total participants, 191 (54\%) were having positive attitude and $163(46 \%)$ were having negative attitude. The study also revealed that $79.7 \%$ were within the poor practice range while $20.3 \%$ showed good practice. Among the demographic variables, area of residence, educational status, and income were significantly associated with mean KAP scores $(\mathrm{P}<0.001)$. A study done in Solomon Islands in 2015 showed that religion was significantly associated with attitude and practice while occupation was only associated with practice and $26.3 \%$ of study participants had good practice. In addition, a study conducted in Bangladesh in 2012 showed that $50 \%$ of study participants had positive.

\section{Objectives}

General objectives: The general objective of the study is to assess the knowledge, attitude and practices on hepatitis B among informal caregivers at Ndola Teaching Hospital.

\section{Specific objectives:}

- $\quad$ To assess the knowledge of HBV infection among informal caregivers at Ndola Teaching Hospital.

- To determine the attitude and practices of informal caregivers towards HBV infection.

- To determine the associations between demographics and knowledge of informal caregivers towards HBV.

- To determine the association between knowledge and attitude of informal caregivers towards HBV.

- To make recommendations to health authorities improve preventive measures against $\mathrm{HBV}$.

\section{Research questions}

- How much do informal caregivers know about hepatitis B?

- What is the attitude of informal caregivers towards hepatitis $\mathrm{B}$ ?

- How is the practice of HBV among informal caregivers

- Is there an association between demographics and knowledge of HBV among informal caregivers?

- Is there an association between knowledge and attitude of HBV among informal caregivers?

\section{Justification of the study}

$\mathrm{HBV}$ is a major problem in Zambia and people suffer and die from complications of the disease daily. To decrease the transmission of HBV in Zambia, it is important to increase the populations' knowledge about the disease, the vaccine and the benefits that comes with it. Review of other studies have shown that the knowledge about $\mathrm{HBV}$ only is average and there are no studies published in Zambia concerning informal caregivers' knowledge, attitude and practices on HBV. Therefore, it is very interesting to carry out this study to investigate the knowledge, attitude and practices on hepatitis $\mathrm{B}$ among informal caregivers at Ndola Teaching Hospital. This is because informal caregivers are seen cleaning and touching the fluid of patients they are looking after without protection (gloves). This act is seen even before the doctors have found the diagnosis making them to be one of the neglected risk population group on HBV. Above all, Ndola Teaching Hospital is the second biggest Hospital in the country and it's the highest referral center on the Northern part of Zambia. The hospital has patients from different parts of Zambia who have different culture and social - economic status. Findings from this study would be very useful in diverse ways, much more it will help reach the WHO goal of eliminating Hepatitis $B$ virus infection by the year 2030 as most people will be aware of the infection and how they can prevent themselves from it.

\section{Measurements}

\section{Operation definitions of concepts:}

- Knowledge-In this study, this is the fact or condition of knowing hepatitis B with familiarity gained through experience or association or other means.

- Attitude-a settled way of thinking or feeling about hepatitis B.

- $\quad$ Practice-in this study, practice means complying or doing preventive measures of acquiring HBV.

- Informal caregiver - is an unpaid family member, friend, or neighbour who provides physical, emotional and often financial support to an individual who is unable to care for him/herself due to illness, injury, disability or other conditions; and needs assistance to manage a variety of tasks, from bathing dressing and taking medications to feeding.

Variables: (Table 1).

\section{Conceptual framework}

(Figure 1). 


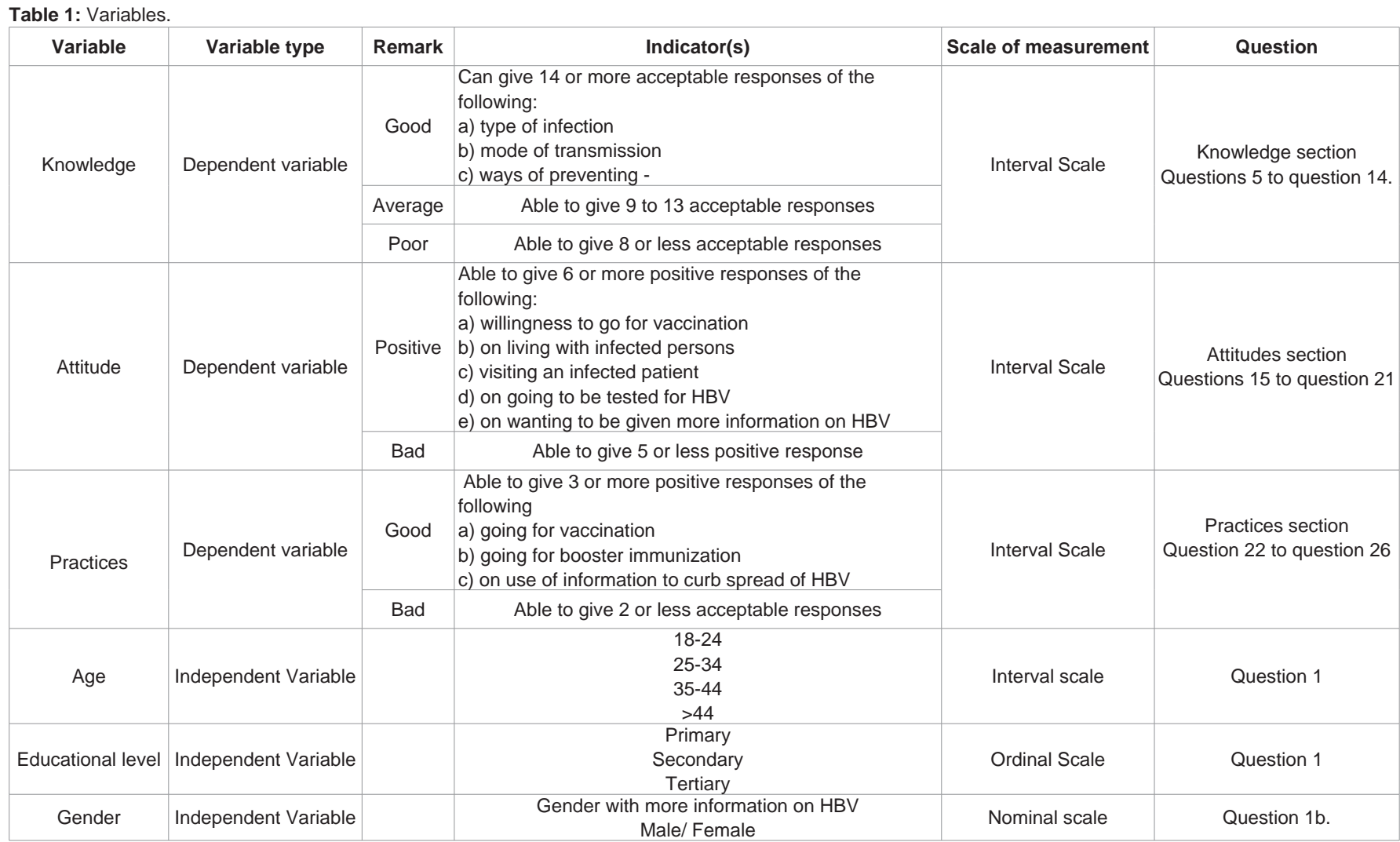

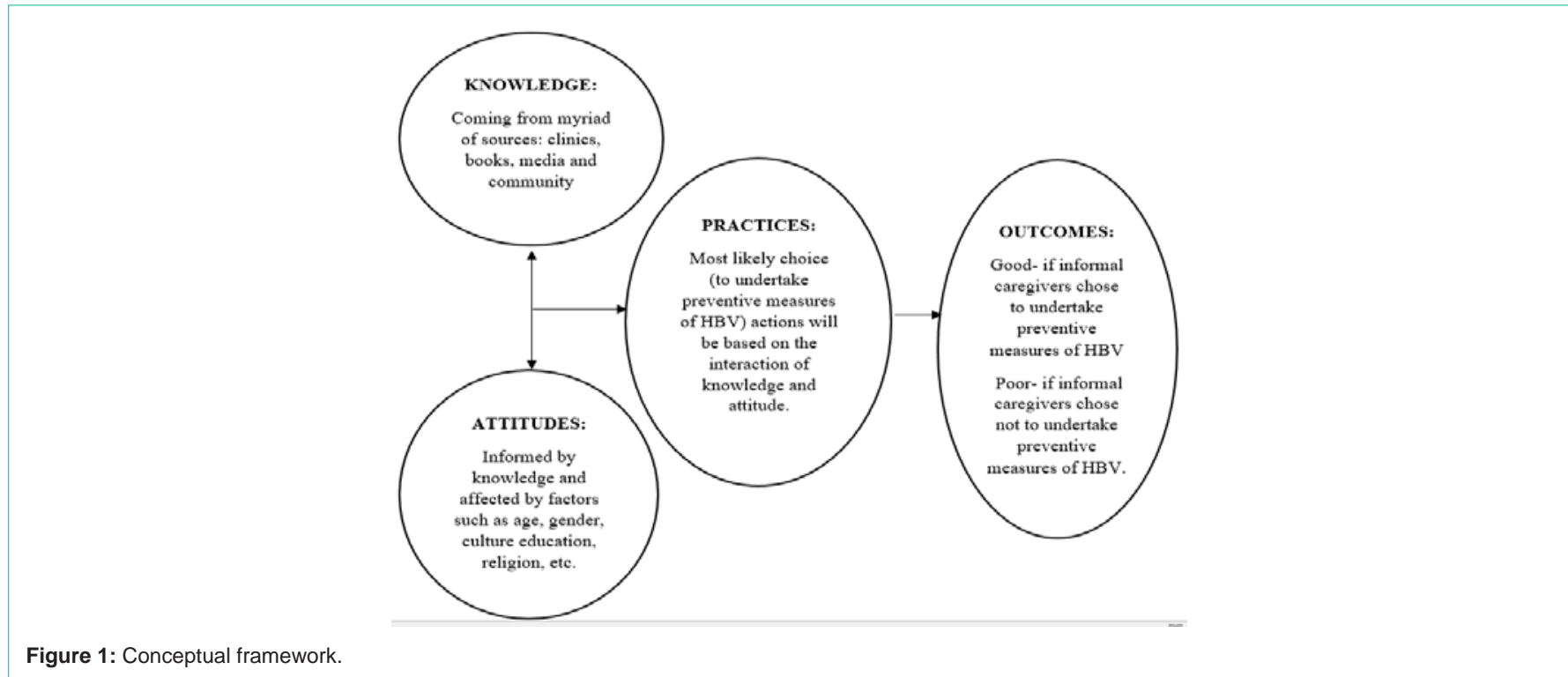

\section{Methodology}

\section{Study site}

The study was done at Ndola Teaching Hospital in Ndola. Ndola city is located approximately $320 \mathrm{~km}$, by road, northwest of Lusaka, the capital and largest city in Zambia. It has Households that fall into middle and low-income groups with a few that fall into high income group. According to ZCSO (2019), Ndola is the third largest city in terms of size and population, with a population of 574,400 , after Lusaka and Kitwe, and second largest in terms of infrastructure development. Ndola Teaching Hospital is the second largest Hospital in Zambia and it is a referral center for the northern part of the country. It has a capacity of 760 active beds.

\section{Target population}

All informal caregivers found waiting for their patients in the three Wards namely; Gynecological ward, Internal medicine wards and surgical wards at Ndola Teaching Hospital were eligible for 
enrolment into the study if they met the inclusion criteria.

\section{Study design}

A non-interventional descriptive, quantitative, study using a cross sectional approach was conducted among the informal caregivers waiting for their patients in Gynecological ward, Internal medicine wards and surgical wards at Ndola Teaching Hospital.

\section{Sample size}

A total of 165 respondents participated in the study. Taking the hospital active bed capacity to be 760 and the three wards of interest to have a total of 336 bed capacity, the sample size calculated was 165 .

The following formula was used:

Samplesize $=\frac{n}{1+\frac{n}{\text { populationsize }}}$

$n=Z^{2} \frac{p(1-p)}{e^{2}}$

$\mathrm{Z}=$ Desired Confidence Interval of $95 \%$

$\mathrm{P}=$ Estimate of the Population Proportion (Prevalence Proportion)

$\mathrm{e}=$ Margin of Error

Using the information in Table 2, the sample size is 165 .

\section{Sampling procedure} bias.

A systematic random sampling was used as it rules out selection

\section{Inclusion and exclusion of the study criteria \\ Inclusion criteria:}

- Informal caregivers at Ndola Teaching Hospital who were waiting for their patients at Gynecological ward, Internal medicine wards and surgical wards

- $\quad$ Both males and females

- Informal caregivers who were 18 years of age and above.

- Those who gave concert to take part in the study.

\section{Exclusion criteria:}

- Non informal caregivers of Ndola Teaching Hospital.

- Informal caregivers who were not from the wards of interest named in the inclusion criteria.

- Informal care givers who were below 18 years of age.

- Those who did not give concert to take part in the study.

\section{Data collection}

Data collection was done using questionnaire-based interviews that were conducted to all participants. To the participants who couldn't understand English, the questionnaire-based interview was done in the language understood by the respondents.

\section{Data analysis}

Data entry, compilations and analysis was done by the use of tables and graphs using SPSS software and Microsoft excel. We conducted univariate analysis and presented results using frequencies and percentages. Associations were calculated in the bivariate analysis using chi-square test of associations to determine associations of interest. Statistical significance was set at 0.05 at 95 percent confidence interval.

\section{Ethical consideration}

Ethical approval was obtained at tropical disease research center. The researcher also got permission from the Copperbelt provincial health office and from Ndola Teaching Hospital management.

The information gathered from this study was strictly confidential. The research information will be disseminated to relevant authorities and with no direct link to participants since anonymity shall be maintained. The study participants were recruited based on their willingness to participate in the study, the information concerning the study was availed to them through a consent form.

\section{Study limitation}

Although this study provides some very useful information on hepatitis B to informal caregivers, the limitations include; the relatively small study population, lack of focused group discussion and in-depth interviews may reduce the transferability of the findings. Further research in this respect could be very useful.

\section{Results}

\section{Demographic characteristics}

A total of 165 informal caregivers from Ndola Teaching Hospital participated in this study. Among the 165 participants, 104 (63.0\%) were females and $61(37.0 \%)$ were males. The highest number of informal caregivers interviewed were aged between 25-34 years of age. The minimum sample age was 18 years. In this study, a clear majority of the participants $(86,52.1 \%)$ had gone as far as secondary level in education. Only $6.7 \%$ had no background of education. Most of the informal caregivers were married (54.5\%) and $6.1 \%$ of the participants were in health related occupation. The above information is shown in Table 3.

\section{Knowledge}

Knowledge scores for individuals were summed to divide the participants into having good knowledge (answered >14/19 knowledge related questions correctly), having average knowledge (answered 9-13 knowledge related questions correctly) and having poor knowledge (who answered $<9$ of knowledge related questions correctly). Status of knowledge among the respondents were summarized in Figure 2. The study findings revealed that of the 165 participants, 88 (53.3\%) had an average knowledge regarding HBV, 39 (23.6\%) had good knowledge and 38 (23\%) had poor knowledge. According to the study findings, HBV was recognized as a viral infection by the majority of the respondents (54.5\%). 68.5\% knew that there was a vaccine for $\mathrm{HBV}$ that can offer protection from acquiring the infection.

Tables 4, 5 and 6 shows the association between age with knowledge, gender with knowledge and educational level with Table 2: Information for Calculating the Sample Size.

\begin{tabular}{|c|c|}
\hline Level of confidence (Z) & 1.96 (at 95\% confidence level) \\
\hline Margin of Error (e) & $5 \%$ \\
\hline Baseline level of the indications (P) & $50 \%$ (as no estimates) \\
\hline
\end{tabular}


Table 3: Socio-demographic profile of informal caregivers.

\begin{tabular}{|c|c|c|c|}
\hline Sr. No. & Particular $(n=165)$ & Frequency & Percent (\%) \\
\hline \multirow{5}{*}{1} & \multicolumn{3}{|c|}{ Age group (in years) } \\
\hline & $18-24$ & 20 & 12.1 \\
\hline & $25-34$ & 73 & 44.2 \\
\hline & $35-44$ & 54 & 32.7 \\
\hline & $>44$ & 18 & 10.9 \\
\hline \multirow{3}{*}{2} & \multicolumn{3}{|c|}{ Gender } \\
\hline & Male & 61 & 37 \\
\hline & Female & 104 & 63 \\
\hline \multirow{5}{*}{3} & \multicolumn{3}{|c|}{ Level of Education } \\
\hline & Primary & 37 & 22.4 \\
\hline & Secondary & 86 & 52.1 \\
\hline & Tertiary & 31 & 18.8 \\
\hline & None & 11 & 6.7 \\
\hline \multirow{3}{*}{4} & \multicolumn{3}{|c|}{ Occupation } \\
\hline & Non health related & 155 & 93.9 \\
\hline & Health related & 10 & 6.1 \\
\hline \multirow{4}{*}{5} & \multicolumn{3}{|c|}{ Marital Status } \\
\hline & Single & 55 & 33.3 \\
\hline & Married & 90 & 54.5 \\
\hline & Divorced/separated & 20 & 12.1 \\
\hline
\end{tabular}

knowledge respectively. Table 4 and Table 5, results shows a not significant relationship ( $\mathrm{p}$ value for age with knowledge was 0,101 and $p$ value for gender with knowledge of HBV was 0.935). However, the $p$ value of education level with knowledge of HBV was $<0.001$ as shown in Table 6. This shows that the association of education level with knowledge of $\mathrm{HBV}$ was very significant. $51.6 \%$ of respondents with good knowledge of HBV had attained tertiary education and only $9.1 \%$ of the respondents with good knowledge had no background of education

\section{Attitudes}

Figure 3 below shows the attitude of informal caregivers. Each Table 4: Association between Ages with Knowledge of HBV.

\begin{tabular}{|c|c|c|c|c|c|c|}
\hline & \multirow{2}{*}{\multicolumn{2}{|c|}{ Variables $(n=165)$}} & \multicolumn{3}{|c|}{ Knowledge of Informal Caregivers } & \multirow{2}{*}{$\begin{array}{l}\text { Chi- square } \\
\text { Test ( } p \text { value) }\end{array}$} \\
\hline & & & \multirow{2}{*}{$\begin{array}{c}\text { Good Knowledge } \\
3\end{array}$} & \multirow{2}{*}{$\begin{array}{c}\text { Average Knowledge } \\
16\end{array}$} & \multirow{2}{*}{$\begin{array}{c}\text { Poor Knowledge } \\
1\end{array}$} & \\
\hline \multirow{11}{*}{ Age } & \multirow{3}{*}{$18-24$} & Count & & & & \multirow{11}{*}{0.101} \\
\hline & & $\%$ within Age & $15.00 \%$ & $80.00 \%$ & $5.00 \%$ & \\
\hline & & $\%$ within Knowledge & $7.70 \%$ & $18.20 \%$ & $2.60 \%$ & \\
\hline & \multirow{3}{*}{$25-34$} & Count & 22 & 32 & 19 & \\
\hline & & $\%$ within Age & $30.10 \%$ & $43.80 \%$ & $26.00 \%$ & \\
\hline & & $\%$ within Knowledge & $56.40 \%$ & $36.40 \%$ & $50.00 \%$ & \\
\hline & \multirow{3}{*}{$35-44$} & Count & 10 & 29 & 15 & \\
\hline & & $\%$ within Age & $18.50 \%$ & $53.70 \%$ & $27.80 \%$ & \\
\hline & & $\%$ within Knowledge & $25.60 \%$ & $33.00 \%$ & $39.50 \%$ & \\
\hline & \multirow[t]{2}{*}{$>44$} & $\%$ within Age & $22.20 \%$ & $61.10 \%$ & $16.70 \%$ & \\
\hline & & $\%$ within Knowledge & $10.30 \%$ & $12.50 \%$ & $7.90 \%$ & \\
\hline
\end{tabular}

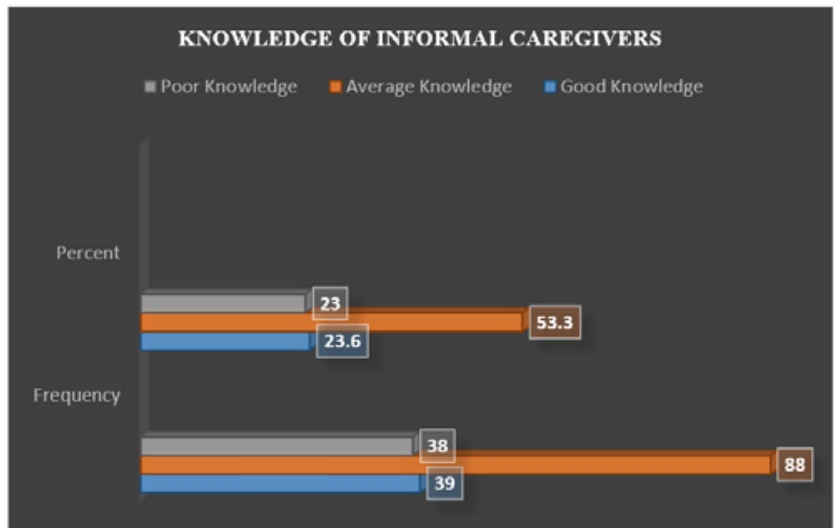

Figure 2: Knowledge levels of informal caregivers towards HBV.

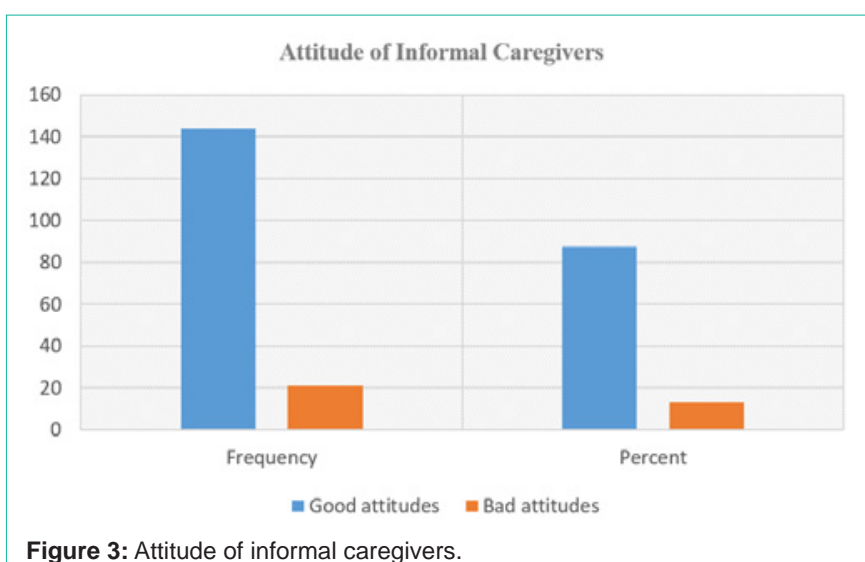

question was labeled with good or bad attitude. A score of 1 was given to good attitude while 0 was given to bad attitude with a score range of maximum of 12 and minimum of 0 . Of the total participants, 144 (87.3\%) of the respondents had good attitudes (answered $\geq 6$ attitude related questions correctly) and only 21 (12.7\%) had bad attitude (who answered $<6$ attitude-related questions correctly). Among the respondents, $84.2 \%$ agreed that they were at risk of acquiring HBV. The respondents who agreed of having no problem in living in the 
Table 5: Association between Genders with Knowledge of HBV.

\begin{tabular}{|c|c|c|c|c|c|c|}
\hline \multirow{2}{*}{\multicolumn{3}{|c|}{ Variables $(n=165)$}} & \multicolumn{3}{|c|}{ Knowledge of Informal Caregivers } & \multirow{2}{*}{$\begin{array}{l}\text { Chi- square } \\
\text { Test ( } p \text { value) }\end{array}$} \\
\hline & & & Good Knowledge & Average Knowledge & Poor Knowledge & \\
\hline \multirow{4}{*}{ Gender of participant } & 促 & Count & 14 & 32 & 15 & \multirow{4}{*}{0.935} \\
\hline & Ivlale & $\%$ within Gender of participant & $23.00 \%$ & $52.50 \%$ & $24.60 \%$ & \\
\hline & \multirow{2}{*}{ Female } & Count & 25 & 56 & 23 & \\
\hline & & $\%$ within Gender of participant & $24.00 \%$ & $53.80 \%$ & $22.10 \%$ & \\
\hline
\end{tabular}

Table 6: Association between Education levels with Knowledge of HBV.

\begin{tabular}{|c|c|c|c|c|c|c|}
\hline & \multirow{2}{*}{\multicolumn{2}{|c|}{ Variables $(n=165)$}} & \multicolumn{3}{|c|}{ Knowledge of Informal Caregivers } & \multirow[t]{2}{*}{$\begin{array}{l}\text { Chi- square } \\
\text { Test ( } p \text { value) }\end{array}$} \\
\hline & & & Good Knowledge & Average Knowledge & Poor Knowledge & \\
\hline \multirow{8}{*}{ Level of education } & \multirow{2}{*}{ Primary } & Count & 6 & 16 & 15 & \multirow{8}{*}{$<0.001$} \\
\hline & & $\%$ within level of education & $16.20 \%$ & $43.20 \%$ & $40.50 \%$ & \\
\hline & \multirow{2}{*}{ Secondary } & Count & 16 & 52 & 18 & \\
\hline & & $\%$ within level of education & $18.60 \%$ & $60.50 \%$ & $20.90 \%$ & \\
\hline & \multirow{2}{*}{ Tertiary } & Count & 16 & 13 & 2 & \\
\hline & & $\%$ within level of education & $51.60 \%$ & $41.90 \%$ & $6.50 \%$ & \\
\hline & \multirow{2}{*}{ None } & Count & 1 & 7 & 3 & \\
\hline & & $\%$ within level of education & $9.10 \%$ & $63.60 \%$ & $27.30 \%$ & \\
\hline
\end{tabular}

Table 7: Association between Knowledge with Attitude of HBV.

\begin{tabular}{|c|c|c|c|c|c|}
\hline \multirow{2}{*}{\multicolumn{3}{|c|}{ Variables $(n=165)$}} & \multicolumn{2}{|c|}{ Attitude of Informal Caregivers } & \multirow{2}{*}{$\begin{array}{l}\text { Chi- square } \\
\text { Test ( } p \text { value) }\end{array}$} \\
\hline & & & Good attitude & Bad attitude & \\
\hline \multirow{6}{*}{ Knowledge of Informal Caregivers } & \multirow{2}{*}{ Good Knowledge } & Count & 37 & 2 & \multirow{6}{*}{$<0.001$} \\
\hline & & $\%$ within Knowledge of Informal Caregivers & $94.90 \%$ & $5.10 \%$ & \\
\hline & \multirow{2}{*}{ Average Knowledge } & Count & 81 & 7 & \\
\hline & & $\%$ within Knowledge of Informal Caregivers & $92.00 \%$ & $8 \%$ & \\
\hline & \multirow{2}{*}{ Poor Knowledge } & Count & 26 & 12 & \\
\hline & & $\%$ within Knowledge of Informal Caregivers & $68.40 \%$ & $31.60 \%$ & \\
\hline
\end{tabular}

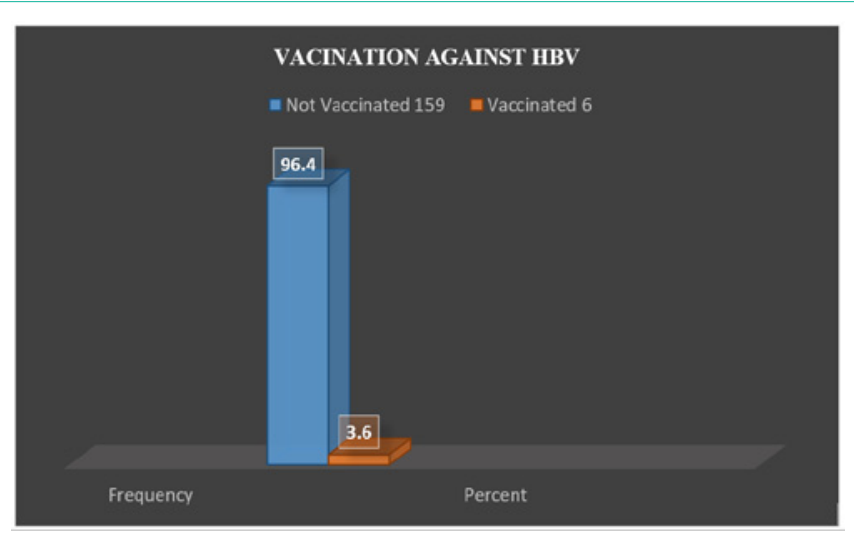

Figure 4: Vaccination against HBV.

same house with a patient with HBV and those who can take care of a HBV patient were $66.7 \%$ and $68.5 \%$ respectively. 121 (73.3\%) were willing to get vaccinated against $\mathrm{HBV}$.

The relationship between knowledge with attitude is shown in Table 7. The study findings showed that the relationship between knowledge with attitude of $\mathrm{HBV}$ was significantly associated
Table 8: Practice of informal caregivers.

\begin{tabular}{|c|c|c|}
\hline \multirow{2}{*}{ Variables $(\mathbf{n = 1 6 5 )}$} & \multicolumn{2}{|c|}{ Practice of Informal Caregivers } \\
\cline { 2 - 3 } & Frequency & Percent \\
\hline Good practice & 0 & 0 \\
\hline Bad practice & 165 & 100 \\
\hline
\end{tabular}

(p-value $<0.001$ ). The findings further highlights that $94.9 \%$ of the informal caregivers with good knowledge of HBV had good attitude and $31.6 \%$ of informal caregivers with poor knowledge of HBV had bad attitude.

\section{Practices}

Each question was labelled with good or bad practice. A score of 1 was given to good practice while 0 was given to bad practice with a score range of maximum of 6 and minimum of 0 . The scale classified practice as good with score $\geq 3$ and bad $<3$. Out of the 165 participants, $165(100 \%)$ showed bad practice. Majority of the respondents 159 (96.4\%) were not vaccinated only $6(3.6 \%)$ were vaccinated against $\mathrm{HBV}$ as shown in Figure 4 and Table 8.

\section{Discussion}

The findings in this current study indicated that knowledge, 
attitude and practices are critical in primary prevention of $\mathrm{HBV}$.

\section{Demographics}

In this study, the majority of informal caregivers who participated were in the age group of 25-34 years (44.1\%) and the majority were females $(63.0 \%)$, similarly in a study done by Strong et al, (2015) the majority of the participants were females (63\%). This shows that most of the informal caregivers are females. Most of the informal caregivers (93.9\%) in this study were not of health related occupation, contrary to most studies on hepatitis B which are done to health workers or students in medical field $[20,23]$. In the current study, $6.7 \%$ informal caregivers had no background of education, this shows that most of the participants in this study had been atleast to a primary school (93.3\%).

\section{Knowledge}

The current findings showed that 88 (53.3\%) of the participants had average knowledge, where as another study conducted on Vietnamese Americans on knowledge and the prevalence of hepatitis B showed that there was a lack of knowledge about HBV, 32.9\% thought incorrectly that $\mathrm{HBV}$ was transmitted through casual contacts or by contaminated air or food. The results also showed that there was a lack of knowledge regarding the treatment, $42.7 \% \mathrm{knew}$ that there was treatment for HBV [18]. Contrary, the current study found that the majority of the respondents 128 (77.6\%) \% knew that there was treatment for HBV and that it is transmitted through blood and fluids.

Another cross- sectional study conducted by Gürakar, Idilman, Keskin and Malik [19] in Turkey reported that $60 \%$ of the participants had basic knowledge that hepatitis B was a transmissible infection and HBV was a major cause of serious liver disease. The findings were similar to the findings of this study as $77 \%$ of respondents in the current study knew that HBV was a major cause of serious liver disease.

The study findings posited that the level of knowledge was not significantly associated with age and gender but it was significantly associated with education level of an individual. A study conducted among nurses and midwives in two maternity hospitals in Khartoum, Sudan on the contrary showed that the level of knowledge was not significantly associated with educational level [21]. The differences may be attributed to the inclusion of a broader range of education level from primary to tertiary in the current study compared to fore mentioned study which focused on nurses and midwives which have all attained tertiary education.

\section{Attitudes}

The results of this study showed that the respondents had good attitude towards hepatitis B (87.3\%) and only $12.7 \%$ of the respondents had bad attitude. In this study $84.2 \%$ of the respondents reported that they knew that they were at risk of contracting HBV by virtue of their work as informal caregivers. $73.3 \%$ were willing to get vaccinated. These findings were similar to the findings of a study done by Mursy et al, [21] which found that the respondents had good attitude (91\%) and $82.7 \%$ were willing to get vaccinated.

The findings of the current study was slightly higher than a study conducted in Ethiopia on pregnant women attending antenatal care at the University of Gondar comprehensive specialized hospital which reported 54\% good attitude [22]. In addition, a study conducted in Bangladesh in 2012 showed that 50\% of study participants had positive attitude.

Having a good attitude means that respondents may be willing to take preventive measures to help combat HBV infection. This willingness was seen in this study as $122(73.3 \%)$ of the respondents wanted to be given information on how HBV is transmitted and $88.4 \%$ wanted information on how the disease can be prevented.

In addition, the study also found that the relationship between knowledge with attitude of HBV was significantly associated (p-value $<0.001$ ). 94.9\% of the informal caregivers with good knowledge of HBV had good attitude and $31.6 \%$ of informal caregivers with poor knowledge of HBV had bad attitude. Promoting awareness of HBV proves be a useful and effective strategy to prevent the spread of HBV, as well as it being a cost-effective strategy to use globally.

\section{Practices}

In this study, it is shown that the respondents had a bad practice of HBV (100\%). 35.8\% had screened for hepatitis B, 3\% received booster immunization against $\mathrm{HBV}$ and only $6(3.6 \%)$ were vaccinated against HBV. The findings were much lower than the findings reported by Gebrecherkos et al., [22] which showed that $20.3 \%$ of study participants had good practice and $85.9 \%$ had not screened for HBV. Another study conducted in Honiara, Solomon Islands, in 2015 showed that $26.3 \%$ of study participants had good practice $[24,25]$.

This bad practice and low number of respondents who are vaccinated in the current study could be as a results of lack of knowledge of respondents on HBV vaccination (60.6\%) and high cost of the vaccine $(50.2 \%) .16 .4 \%$ of the respondents feel it is not necessary to wear groves when taking care of the patient and to get vaccinated.

The findings of this study need to be considered in the context of some limitations; this is a cross-sectional study done in one site, which might limit results generalization for all settings in the country. Further analysis is needed to investigate risk factors that influence KAP level of informal caregivers towards HBV infections.

\section{Conclusion}

The result of this study showed the majority of the participants had average knowledge on hepatitis B virus. A statistical significant difference could not be shown between the genders for the level of knowledge and between ages with knowledge. However, there is a close relationship between education levels with the level of knowledge of HBV. The results of the informal caregivers' attitude towards hepatitis B was very good and more than a third quarters of the participants had a score of ten to eleven out of twelve in the attitude part of the questionnaire. The result showed a significant difference between the knowledge with attitude. Those who had knowledge had good attitude too. The study reveals a low level of HBV vaccination coverage and the practice aspect of HBV was generally bad and this was as a result of respondents not knowing where to get a vaccine, the high cost of these vaccine and others had no knowledge about how to fully protect oneself from acquiring HBV. Therefore, improving the 
knowledge about HBV can contribute to improve the public health by getting lower rate of cases with HBV

\section{Recommendations}

Hepatitis B is a major health problem globally casting an enormous burden on the health-care system a major source of patient's misery. This illness or disease is preventable. The author therefore recommends that following this timely study primary prevention approach be taken by extensively and frequently teaching all informal care givers in all hospitals and clinics on how to protect themselves from the disease.

Pictorial Posters depicting the proper way of handling patients by informal caregivers be displayed in health centers (wards and OPDs) and public posters in as simple and comprehensible language as possible should be used for easy learning. Community based educational talks should be conducted on a routine basis by community-based health volunteers.

Furthermore, gloves should be offered to all informal caregivers especially the primary informal caregivers who have a direct contact with their patients and the government should ensure that vaccinations are done in all hospitals at an affordable price for all citizens.

\section{Acknowledgement}

I wish to first and foremost thank God for his love and faithfulness throughout my research journey. I am also indebted to my supervisor Mr. Eric Njunju for his tireless dedication and effort in seeing to it that this thesis is successfully and timely completed. Extreme special thanks also go to my co-supervisor, the Public health lecturer, Dr. David Mulenga for creating a fresh and stress free academic atmosphere through the regular supervisions and guidance made that saw to the completion of this thesis. His efforts and tireless dedication to this piece of work will always be appreciated. I would also wish to show my gratitude to Mr and Mrs Siamunyanga for the ever present and tireless financial and emotional support. Lastly, I'm indebted to the support and advice of my friends and classmates.

\section{Dedication}

This research is dedicated to My Beloved Parents Sophia Siambulo and Millias Nyeleti, my two brothers Clifford and Kibbick and my lovely four sisters Milliam, Rhoidah, Luster and Felisters.

\section{References}

1. World Health Organization. Immunization, vaccines and biological, hepatitis B. 2012.

2. Weinbaum CM, Mast EE, Ward JW. Recommendations for identification and public health management of persons with chronic hepatitis B virus infection. Hepatology. 2009; 49: 35-44

3. Liang TJ. Hepatitis B: The Virus and Disease. Hepatology (Baltimore, Md.). 2009; 49: S13-S21.

4. Glebe D, Konig A. Molecular virology of hepatitis B virus and targets for antiviral intervention. Intervirology. 2014; 57: 134-140.
5. World Health Organization. Hepatitis B vaccines. Weekly epidemiological record. 2009; 84: 405-419.

6. World Health Organization. Hepatitis B. 2017.

7. Mayoclinic.org. Hepatitis B Causes-Mayo Clinic. 2016.

8. ZAMPHIA. Zambia Population-based HIV Impact Assessment report. 2016.

9. NHSP. National Health Strategic Plan. 2017; 2017-2021.

10. Blumberg BS, Gerstley BJS, Hungerford DA. et al. A serum antigen (Australia antigen) in Down's syndrome, leukemia and hepatitis. Ann. Internal Med. 1967; 66: 924-931.

11. Dane DS, Cameron $\mathrm{CH}$, Briggs M. Virus-like particles in serum of patients with Australia-antigen-associated hepatitis. Lancet. 1970; 10: 695-698.

12. Dunford L, Carr MJ, Dean J, Nguyen LT, Hall WW. A multicenter molecular analysis of hepatitis $B$ and blood-borne virus coinfections in vet nam. pLos one. 2012; 7: e39027.

13. Rossi C, Shrier I, Marshall L, Cnossen S, Schwartzman K, Greenaway C. Seroprevalence of chronic hepatitis B virus infection and prior immunity in immigrants and refugees: a systematic review and meta-analysis. PLos one. 2012; 7: e44611.

14. World Health Organization. Hepatitis B. 2015.

15. Centers for Disease Control and Prevention. Viral Hepatitis - Hepatitis B information. Atlanta: Centres for Disease Control and Prevention. 2015.

16. Hepatitis B Foundation. Hepatitis B and Primary Liver Cancer. Hepatitis B Foundation. 2014.

17. Marinho R, Meireles L, van Damme P. Three decades of hepatitis B contro with vaccination. World Journal of Hepatology. 2015; 7: 2127-2132.

18. Bui C, Ha N, Leduc T, Nguyen M, Nguyen T, Trinh H, et al. Prevalence Risk Factors, and Disease Knowledge of Chronic Hepatitis B Infection in Vietnamese Americans in California. Journal of Cancer Education. 2013; 28: 319-324.

19. Gürakar M, Idilman R, Keskin O, Malik M. Public awareness of hepatitis B infection in Turkey as a model of universal effectiveness in health care policy. Turkish Journal of Gastroenterology. 2014; 25: 304-308.

20. Buisson $Y$, Latthaphasavang $V$, Khampanisong $P$, Pathoumthong $K$, Souvong $V$, Quet F. Vaccination status, knowledge and awareness towards hepatitis B among students of health professions in Vientiane, Lao PDR. Vaccine. 2014; 32: 4993-4999.

21. Mursy SMM, Mohamed SOO. Knowledge, attitude and practice towards hepatitis $B$ infection among nurses and midwives in two maternity hospitals in Khartoum, Sudan. BMC Public Health. 2019; 19: 1597.

22. Gebreneskel T, Beshah T, Tesfaye M, Beletew B, Mengesha A, Getie A. Assessment of knowledge and practice on hepatitis $B$ infection prevention and associated factors among health science students in Woldia University, Northeast Ethiopia. Advances in preventive medicine. 2020; 2020: 9421964

23. Carter T, Damico A, DeHart M, Halsey N, Marcuse E, Mergler M, et al. Association of vaccine related attitudes and beliefs between parents and health care providers. Vaccine. 2013; 31: 4591-4595.

24. The National Board of Health and Welfare. Patientens Delaktighet. Stockholm: Socialstyrelsen. 2011.

25. UNAIDs U, World Health Organization. Global HIVIAIDS response: epidemic update and health sector progress towards universal access: progress report 2011. Global HIVIAIDS response: epidemic update and health sector progress towards universal access. 2011. 